\title{
Thanks to all those who reviewed for Journal of Medical Case Reports in 2015
}

Michael R. Kidd

\section{Contributing reviewers}

A peer-reviewed journal would not survive without the generous time and insightful comments of the reviewers, whose efforts often go unrecognized. Although final decisions are always editorial, they are greatly facilitated by the deeper technical knowledge, scientific insights, understanding of social consequences, and passion that reviewers bring to our deliberations. For these reasons, the Editor-in-Chief and staff of the Journal of Medical Case Reports warmly thank the 708 reviewers whose comments helped to shape the journal, for their invaluable assistance with review of manuscripts in Volume 9 (2015).

Maria Ida Abbate

Italy

Ahmad Abdelkarim

USA

Mahmoud Abdelsalam

USA

Eman Abdelzaher

Egypt

Hassan Abduljabbar

Saudi Arabia

\section{S Abrassart}

Switzerland

Arun AC

India

Mulat Adefris

Ethiopia

Gauhar Afshan

Pakistan

Maryam Afshar

USA

Amitesh Agarwal

USA

\author{
Puneet Aggarwal \\ India \\ William Agger \\ USA \\ Varun Agrawal \\ USA
}

\section{Abdul Razzaque Ahmed \\ USA}

Fouad Al- Bayaty

Malaysia

Badr Al-Bawardy

USA

Bakr Alhussaini

Saudi Arabia

Abdullah Alhwiesh

Saudi Arabia

Yasser Al-Jehani

Saudi Arabia

Laith Alrubaiy

UK

Mohammad Al-Sabi

Denmark
Lisa Amir

Australia

Theodore Anagnostou

Greece

Olympia Anastasiou

Germany

Edwin J Anaya-Pava

Mexico

Mario Angelico

Italy

Alessandro Antonelli

Italy

Suresh Antony

USA

\section{Stylianos Apostolidis}

Greece

\section{Alexandros Apostolopoulos}

Greece

Edward Araujo Júnior

Brazil

Ripudaman Arora

India

Correspondence: michael.kidd@flinders.edu.au

Faculty of Health Sciences, Flinders University, Adelaide 5001, SA, Australia 
Hammad Arshad

USA

Irene Asouhidou

Greece

Adolfo Attili
Italy

Awoniyi Awonuga

USA

Georgios Ayiomamitis

Greece

Irfan Ayub
India

Joseph Azuri

Israel

Dayanand Bagdure

USA

Anne Bakker

Netherlands

Nikolaos Baltayiannis

Greece

Bernardo Barahona-Correa

Portugal

Roberta Barbera

Italy

Kelli Barbour

USA

Alécio Barcelos

Brazil

Bilal Barkatali

UK

Giancarlo Barraco

Italy

Curtis Barry

USA

Özge Basaran

Turkey

Dan Bates

Australia

Himmatrao Bawaskar

India

Huseyin Bayazit

Turkey

Jasmina Begum

India
T Z Belhocine

France

Ahmed Belkouch

Morocco

Shannon Beres

USA

Peter Berlit

Germany

Christophe Berney

Australia

Alvise Berti

Italy

Rajesh Bhagat

USA

Rajesh Bhagat

USA

Sanjay Bhatia

USA

Dave Bhattacharya

USA

Anirban Bhattacharyya

USA

Debesh Bhoi

India

Zbigniew Binienda

USA

Rajshekhar Bipeta

India

Yochai Birnbaum

USA

Einar Bjornsson

Iceland

Miguel Blanca

Spain

Joerg Blessmann

Germany

Agnes Bloch-Zupan

France

Benoit Blondeau

USA

Benoit Blondeau

USA

Raphael Bodensohn

Germany
Jean-Jacques Boffa

France

Marianne Böni-Schnetzler

Switzerland

Kacy Bonnet

USA

Italo Borini

Italy

Christopher Boston

USA

Luca Botta

Italy

Stephen Boyle

Australia

Patrick Bradley

UK

Manjunatha BS

India

Sebina Bulic

USA

Klaus Burkhart

Germany

Larry Bush

USA

Mehmood Butt

UK

Cen Bytyqi

Albania

Carlo Caffarelli

Italy

Mario Camarda

Italy

Mehmet Akif Camkurt

Turkey

Giuseppina Campisi

Italy

David Cantu de Leon

Mexico

Joshua Carothers

USA

Jose Carreno

Colombia

Vincenzo Caruso

UK 
Santos Castañeda

Spain

Roberto Casula

UK

Marco Cei

Italy

Bryan Chai

USA

Anthony W H Chan

Hong Kong

Yoon-Seok Chang

Korea, South

Woohyok Chang

Korea, South

Seho Chang

Korea, South

Arjun Chanmugam

USA

Ronnie Chee

UK

Jun Chen

China

M Chen

China

Y C Chiang

Taiwan

Toshimi Chiba

Japan

Joseph Choukroun

France

Stefano Cianetti

Italy

J Ciccolini

France

Grzegorz Cieslar

Poland

Frédéric Clarençon

France

William Clark

Canada

Yehuda Cohen

USA

Zachary Corbin

USA
Zachary Corbin

USA

Raul Corrales

Chile

Maria Lucia Correa-Giannella

Brazil

Anna Crocco

Italy

Sara Cruz

Portugal

Halisson Cruz

Brazil

Banushree CS

India

Kenji Cunnion

USA

M Cusidó

Spain

Hanady Daas

USA

Rongping Dai

China

Johanna Daily

USA

Giovanni Damiani

Italy

Adebiyi Damola

UK

Cem Dane

Turkey

Sambhunath Das

India

Krishanu Das

India

Fabrizio D'Ascenzo

Italy

Nandini Dave

India

Arka De

India

Chandima de Alwis

Australia

Guilherme de Jesus

Brazil
Marcelo Bezerra de Menezes

Brazil

Alexander Dechêne

Germany

Srihari Deepak

UK

Archie Defillo

USA

Hakkı Değer

USA

D Demellawy

Canada

Nihat Demir

Turkey

Rafael Denadai

Brazil

Omur Dereci

Turkey

Daniel Deschler

USA

Daniela Di Venere

Italy

Georgios Dimitrakakis

UK

Ioannis Dimitroulis

Greece

Charles Dinarelo

USA

Ramakant Dixit

India

Frank Domino

USA

Sarah Donaldson

USA

Marc Donath

Switzerland

Dimitrios Dougenis

Greece

Dimitrios Dragoumis

UK

Stamatoula Drakopoulou

Greece

Georgios Drosos

Greece 
Ravi Durvasula

USA

Lee Eckhardt

USA

Hawa Edriss

USA

Ghada El Sagheer

Saudi Arabia

Ahmed El-Assmy

Egypt

Stephanie Elkins

USA

Adel El-Naggar

USA

Irene Esposito

Germany

Giuseppe Maria Ettorre

Italy

Mehtap Evran

Turkey

D Faas

Germany

Lee Fairlie

South Africa

Dimitrios Farmakiotis

USA

Francesca Farnetani

Italy

Hasan Fattah

USA

Stefano Fedele

UK

Steve Fenton

USA

Luis Fernández Salazar

Spain

Leslie Fiengo

UK

Anneliese Fischer Thom

Brazil

Joseph Fokam

Cameroon

Francois Fong

Hong Kong
T J Forbes

USA

Evangelia Fouka

Greece

Renato Franco

Italy

Laura Franco

Italy

Mary Fristad

USA

Ho Gwo Fuang

Malaysia

Clotilde Fuentes-Orozco

Mexico

Takaaki Fujii

Japan

Takashi Fujita

Japan

Takahiko Fukuchi

Japan

Koichi Fukunaga

Japan

Ioannis Galanis

Greece

Giovanni Galati

Italy

Danijel Galun

USA

Maurizia Gambacorta

Italy

Anthony Gannon

USA

Ann Gardner

Sweden

Bhavuk Garg

India

Rakesh Garg

India

Vishnu Garla

India

Roberto Gatto

Italy

Nurperi Gazioglu

Turkey
Marco Gazzoni

Italy

Ivan Gentile

Italy

Kemel Ahmed Ghotme Ghotme

Colombia

Stilianos Giannakopoulos

Greece

Kleanthis Giannoulis

Greece

Shah Giashuddin

USA

Rodney Gilbert

UK

Matteo Giorgi Pierfranceschi Italy

Maria Gkika

Greece

Simona Glasberg

Israel

Jeroen Goede

Switzerland

Atul Goel

India

Elizabeth Golesic

Canada

Sripad Gopalakrishna Mehandale USA

Ganesh Gopalakrishnan

India

David Gorard

UK

Karen Gordon

UK

Steven Goudy

USA

Lajya Goyal

India

Tarang Goyal

India

Barbara Gracious

USA

Josep M Grau

Spain 
Andrew Green

Ireland

Adi Greenberg

Israel

Neil Greening

UK

Alistair Grey

UK

David Griffith

USA

Paraskevas Grivas

Greece

Gerald Gruber

Austria

Paolo Guarascio

Italy

W Gudu

Ethiopia

Nazmi Gultekin

Turkey

Deepak Gunjan

India

Gaurav Gupta

USA

Nitin Gupta

India

D K Gupta

UK

Anju Gupta

India

Vijay Hadda

India

Takahiro Haga

Japan

Christlieb Haller

Switzerland

Tsukasa Hanemoto

Japan

Guy Hans

Belgium

Noboru Hara

Japan

Kiyoshi Harada

Japan
Uma Hariharan

India

Leanne Harling

UK

Aoife Healy

UK

Tomás Henlín

Czech Republic

Marion Henry

USA

Manuel Hernandez-Guerra

Spain

Sarah Heron

Australia

Jesica Herrick

USA

Farhad Heydarian

Iran

Catherine Hill

Australia

Melissa Hilmes

USA

Konrad Hoetzenecker

Austria

Johannes Hofer

Austria

Shuji Horibe

Japan

Hironobu Hoshino

Japan

Dingming Huang

China

Caren Hughes

USA

Nexhmi Hyseni

Kosovo

Akihiko Ikeda

Japan

Munir Iqbal

Canada

Ahaorvi Isa

Canada

Mitsuaki Ishida

Japan
Hideya Ito

Japan

Ali Izadpanah

Canada

Hossain Jabbari

Austria

Janet Jacobson

USA

Yves Jacquemyn

Belgium

Sujit Jagtap

India

Vivek Jain

UK

Neetu Jain

India

Praveen Jajoria

USA

Bawo James

Nigeria

Vijayakumar Javalkar

USA

Hemangi Jerajani

India

Kathie-Ann Joseph

USA

Bharti Joshi

India

Milind Joshi

India

Matthias Jürgens

Germany

Stefanos Kachrilas

United Arab Emirates

Korhan Kahraman

Turkey

H Kaimakliotis

USA

Nikolaos Kalinderis

Greece

Kivanc Kamburoglu

Turkey

Kazuaki Kameda

Japan 
Gokul Vignesh Kanda Swamy UK

\section{Takahiro Kanno}

Japan

Paige Kaplan

USA

Neha Kapoor

UK

Levent Kaptanoglu

Turkey

Rie Karasawa

Japan

Manmeet Kaur

USA

Leticia Kawano-Dourado

Brazil

Joelle Kefer

Belgium

Jessica Keim-Malpass

USA

Martina Kelly

Canada

Y Keren

USA

Isaak Kesisoglou

Greece

Mohamed Kharfan-Dabaja

USA

Mahmoud Khattab

Egypt

Ayaz Khawaja

USA

Divya Khosla

India

Eva Kieslichová

Czech Republic

Wook Kim

Korea, South

Kumiko Kimura

Japan

Sandor Klis

Netherlands

Celebi Kocaoglu

Turkey
Christian Koch

USA

Maurizio Koch

Italy

Daniel Kokong

Nigeria

M K Kolar

UK

Likurgos Kolilekas

Greece

Eija Könönen

Finland

Z Korzets

Israel

Jan Kotarski

Poland

Kalliopi Kotsa

Greece

Vasileios Kouranos

Greece

Ioannis Koutelidakis

Greece

Gopal Kowdley

USA

Tomohide Koyama

Japan

Ali Koyuncuer

Turkey

Somasekhar Krishna USA

Karen Krogfelt

Denmark

Bindu Kulshreshtha

India

Priya Kumthekar

USA

Jujju Kurian

India

Ali Kurt

USA

Andreas Kurtz

Germany

Kathryn Kvam

USA
Bernice Kwong

USA

Dimitrios Kyparissopoulos

UK

Stephen E Lapinsky

Canada

José Luis Lázaro Martínez

Spain

Milan Lepic

Serbia

Aaron Lesher

USA

Claudio Letizia

Italy

Roland Leung

Hong Kong

Steven Lewis

USA

Philippe Lheureux

Belgium

Bo Li

Canada

Zhiming Li

China

Gordon Li

USA

Chien-Yu Lin

Taiwan

Kuan-Chou Lin

Taiwan

Katerina Linhartova

Czech Republic

Michael Linnebank

Switzerland

Jules Lipoff

USA

Jing Liu

USA

Steven Livesey

UK

Lorenzo Lo Muzio

Italy

Martín Lorenzo

Spain 
Marta Losa Iglesias

Spain

Peter Lu

Singapore

Chin-Fang Lu

Taiwan

Gilberto Luna Lugo

Mexico

Jicheng LV

China

Sally Ann Lynch

Ireland

Ian Mackenzie

UK

Karan Madan

India

Brett Mador

Canada

Marc Maegele

Germany

Gaetano Magro

Italy

Anand Mahadevan

USA

Ashoka Mahapatra

India

Mohamad Mahmoud

USA

Michael Mahmoudi

UK

Matthias Maiwald

Singapore

Chloe Mak

Hong Kong

Vijay Malpathak

India

Effrosyni Manali

Greece

Ayse Tulin Mansur

Turkey

Nizar Maraqa

USA

Dan Marek

Czech Republic
Athanasios Marinis

Greece

Konstantinos Markou

Greece

Henri Marret

France

Ian Marshall

Australia

Thomas Marth

Germany

Haruhiko Maruyama

Japan

Helena Marzo-Ortega

UK

Gwinyai Masukume

Zimbabwe

Joseph Kunju Mathew

India

Ranjana Mathur

Singapore

Shinya Matsuzaki

Japan

Valeria Mazzi

Italy

Federico Mecacci

Italy

Bruno Megarbane

France

Markus Meissner

Germany

Renuka Melkundi

India

Moises Mercado

Mexico

Elif Meseci

Turkey

Ahmed Messallam

USA

Linda Metaxa

Italy

Pavel Michalek

Czech Republic

Antonios Michalopoulos

Greece
Nickolaos Michalopoulos

Greece

George Michalopoulos

Greece

Ioannis Mikoniatis

Greece

Jeronimo Milano

Brazil

Kostas Mimidis

Greece

Bushra Mina

USA

Alireza Minagar

USA

Ravinder Minhas

India

Tammy Mirensky

USA

Hidekazu Misaki

Japan

Georgios-Alexandros Mistriotis

UK

Jun Miyauchi

Japan

Consuelo Modesto

Spain

Kathryn Moffett

USA

Mohamed Ali Ahmed Mohamed Egypt

Amr Mohamed

UK

Shahida Mohd-Said

Malaysia

George Moll

USA

Guillermo Monsalve

Colombia

Tom Moreels

Belgium

Akira Morimoto

Japan

Naoto Morimura

Japan 
Michael Moritz

USA

Karwan Moutasim

UK

Theodoros Moysidis

Germany

Nicola Mumoli

Italy

Zeynel Mungan

Turkey

William Murk

USA

Michelle Murray

Ireland

Natalia Myakova

Russian Federation

Eleni-Aikaterini Nagorni

Greece

Satoshi Nagoya

Japan

Manu Nair

UK

K Naito

Japan

R Nambron

USA

Tsutomu Namikawa

Japan

Abdulrasheed Nasir

Nigeria

Ramon Navarro

United Arab Emirates

Rodrigo Navarro-Ramirez

Mexico

Umit Nayki

Turkey

Ujjwal Neogi

Sweden

Nnabuike Chibuoke Ngene

South Africa

Peggy Nguyen

USA

Yuzuru Niibe

Japan
Hiroaki Nishioka

Japan

Xiaohui Niu

China

Joseph NM

India

Francisco Nogales

Spain

Maria Claudia Nogueira Zerbini

Brazil

Takehiro Noji

Japan

Kandai Nozu

Japan

Dimitrios Ntelios

Greece

Achilleas Ntinas

Greece

Anthony Nyamache

Kenya

F Ochsendorf

Germany

Onyema Ogbuagu

USA

Aung Oo

UK

Norberto Ortego-Centeno

Spain

Eleonora Ortu

Italy

Zdenko Ostojic

Bosnia and Herzegovina

Louise Pacher Hoffmann

Brazil

Vinesh Padayachy

South Africa

Sukhmani Padda

USA

Otávio Pagin

Brazil

Yatan Pal

USA

Ajay Pal Singh

India
Periklis Panagopoulos

Greece

Ravinder Pandey

India

Vasileios Papadopoulos

Greece

Kostas Papagiannopoulos

UK

Nikolaos Papanas

Greece

Theodossis S Papavramidis

Greece

Spyros Papiris

Greece

Yael Paran

Israel

Jin Kyun Park

Korea, South

Nigel John Parr

UK

Valentina Pastore

Italy

Athanasia Pataka

Greece

Jigisha Patel

UK

Konstantinos Paterakis

Greece

Sandip Pati

USA

Anil Patki

India

John Pauling

UK

S Paydas

Turkey

Deniz Peker

USA

Erdal Peker

Turkey

Brenton Pennicooke

USA

Judith Pentz

USA 
Katy Peters

USA

Paul PG

India

Andrea Phillipou

Australia

Adriano Piattelli

Italy

Giorgina Barbara Piccoli Italy

Michael Pitiakoudis

Greece

Constantinos Pitsios

Greece

Roy Poblete

USA

Andreas Polydorou

Greece

Mike Polyzonis

Greece

Anna Potulska-Chromik

Poland

Harry Powell

UK

Mukhyaprana Prabhu

India

Antonino Michele Previtera

Italy

Xingshun Qi

China

You-Wen Qian

USA

Waris Qidwai

Pakistan

Stuart Quan

USA

Keerthi Ramesh

India

Leonardo Ramirez Arreola

Mexico

Sahaj Rathi

India

Sahaj Rathi

USA
Amir Ravandi

Canada

Daniel Raymond

USA

P Reper

Belgium

Benjamin Rhodes

UK

Susan Richardson

Canada

Daniel Riche

USA

John Ringman

USA

Richard Rison

USA

Nicoletta Riva

Italy

Sandro Rizoli

Canada

Michele Roberts

USA

Paul Robinson

UK

Raffaele Rocchi

Italy

Manuel Rodriguez-Davalos

USA

Udo Rolle

Germany

Ilaria Romani

Italy

Damian Romero

USA

Lorraine Rosamilia

USA

Barry Rosenbloom

USA

Lionel Rostaing

France

Jessica Roybal

USA

Mourelatou Roza

Greece
Andres Mariano Rubiano Escobar

Colombia

Vidar Ruddox

Norway

Antonio E Ruiz Serrato

Spain

Jaime Ruiz -Tovar

Spain

Clark Russell

UK

Durre Sabih

Pakistan

Ahmed-Ramadan Sadek

UK

Ali Sadrizadeh

Iran

Arun Saini

USA

Atsushi Sakuraba

USA

Rosa Salazar

Spain

Nikolaos Salemis

Greece

Sundeep Saluja

India

Jayanta Samanta

India

Nicolas Sananes

France

Mario Santini

Italy

Vidyadhar Sardesai

India

Shinya Sato

Japan

Paola Savoia

Italy

Ilyas Sayar

USA

Francesco Sbrana

Italy

Bernhard Schaller

UK 
Bernhard Schaller

France

Richard Scher

USA

Ellen Scherl

USA

Breno Schor

Brazil

Jan Schroeder

Italy

Cara Sedney

USA

Marta Seghieri

Italy

Virendra N Sehgal

India

Ali Seifi

USA

Francis Seow-Choen

Singapore

David Seres

USA

Arnold Seto

USA

Utkan Sevuk

Turkey

Daniel Alexander Shaerf

UK

Daniel Shaerf

UK

Lokesh Shahani

USA

Lee Shapiro

USA

Alka Sharma

India

Shilpa Sharma

India

Aparna Sharma

India

Shakti Sharma

India

Dominick Shaw

UK
Susan Shaw

USA

Manjunath Shenoy

India

Jiangang Shi

China

Nektaria Sidiropoulou

Greece

Matthew Siedhoff

USA

Navneet Singh

India

D A Sippo

USA

Panagiotis Skendros

Greece

Thomas Skripuletz

Germany

Samantha Sloan

UK

Stuart Smith

UK

Ioannis Sokolakis

Greece

Jean-Karl Soler

Malta

Cristina Solomon

Austria

Amey Sonavane

India

Gianluca Sottilotta

Italy

Vasileios Souftas

Greece

Karen Speck

USA

John Spurzem

USA

Thambipillai Sri Paran

Ireland

James Stefanou

UK

Paschalis Steiropoulos

Greece
Sara Stern

USA

Jose Subauste

USA

Anbukkani Subbian

India

Koichiro Sugimura

Japan

Tae-Yun Sung

Korea, South

Bhaskar Suryanarayanan

India

Akio Suzumura

Japan

Mathias Tiedemann Svendsen

Denmark

Yechiel Sweed

Israel

Clodagh Sweeney

Ireland

Brian Swick

USA

Federico Tacconi

Italy

Guillaume Taieb

France

Arturo Tamayo

Canada

Bien Keem Tan

Singapore

Yoshinari Tanaka

Japan

Giovanni Tarantino

Italy

Phil Taussky

USA

Marco Teli

Italy

Irene Terzi

Greece

Sidney Tessler

USA

Jagdeep Thakur

India 
Loukas Thanos

Greece

Mark Thomas

New Zealand

Nicholas Todd

UK

Yukiharu Todo

Japan

Esteban Torche

Chile

Paschalis Tossios

Greece

Silvia Trifiro

Italy

Stavros Tryfon

Greece

Athina Tsili

Greece

Bernard Tuch

Australia

Mutahir Tunio

Saudi Arabia

Vasilios Tzilas

Greece

A Udelnow
Germany

M Iftekhar Ullah

USA

Anayat Ullah

UK

Nurcan Unver

Turkey

Vijai Upadhyaya

India

Shinji Urakami

Japan

Nicolás Useche

Colombia

Sinan Uzman

Turkey

Kamen Valchanov

UK

Alejandra Valenzuela

USA
Virginia van Duyne

USA

Robert-Jan van Geuns

Netherlands

Ivor Vanhegan

UK

Vittorio Vanini

Italy

John Vaughn

USA

Anand Venkatraman

USA

Giovanni Vennarecci

Italy

Abhai Verma

India

Serena Vettori

Italy

Kenneth Vick

USA

Nicolas Vignier

France

Kumar Vijay

India

Roberto Villani

Italy

Ana Paula Vivas

Brazil

Mavroudis Voultsos

Greece

Stergios Vradelis

Greece

Spyridon Vrakas

Greece

Zoran Vratnica

Montenegro

Sidhesh Wagh

India

Heather Wakelee

USA

Mohammad Sami Walid

USA

Elaine Walsh

Ireland
Stephen Walsh

USA

Zhongxiao Wang

USA

Imtiaz Wani

India

Orli Wargon

Australia

Toru Watanabe

Japan

Laura Waters

UK

Rafal Watrowski

Germany

Katrin Weier

Canada

Huang Wen-Bin

China

Sandra Werner

USA

Elspeth Whitby

UK

Jill Whitehouse

USA

Timothy Wiegand

USA

Michael Winkelman

Brazil

Grace Wong

Hong Kong

X Wu

China

Daisuke Yabe

Japan

Sachin Yallappa

UK

Satoru Yamada

Japan

Sohsuke Yamada

Japan

Yan Yan

China

Toru Yanagawa

Japan 
Huilin Yang

China

Bienvenido Yangco

USA

Yukihiro Yano

Japan

Danielle Yanuck

USA

Mohamed Yassin

USA

Thomas Yau

Hong Kong
Edward Young

USA

Selcuk Uksel

Turkey

Sadik Yurttutan

Turkey

Zacharias Zachariah

Switzerland

Marco A Zanini

Brazil

Paul Zarogoulidis

Greece
Jianquan Zhang

China

Ling Zhang

USA

Dongsheng Zhou

China

Vas Zochios

UK

Abdolali Zolghadrasli

Iran

Alauldeen Zubair

Iraq 